Journal of Advanced College of Engineering and Management, Vol. 3, 2017

\title{
PROPERTIES RELATED TO WEIGHT FUNCTION
}

\author{
Vinod Parajuli ${ }^{1}$ and Santosh Ghimire ${ }^{2}$ \\ ${ }^{1}$ Assoc.Professor, Department of Science \& Humanities, IOE, Pulchowk Campus, T.U. \\ Email Address:vparajuli@hotmail.com \\ ${ }^{2}$ Department of Science \& Humanities, IOE, Pulchowk Campus, T.U. \\ Email Address: santoshghimire@ioe.edu.np
}

\begin{abstract}
In this paper, we begin with brief discussion of theory of weights and $A_{p}$ weight functions. We then state and prove some of the properties of $\mathrm{A}_{\mathrm{P}}$ weight function using elementary analysis tools.
\end{abstract}

Key words: $A_{1}$ weight function, Maximal functions, $A_{p}$ weight function, Holder's Inequality.

\section{Introduction}

The theory of weight are useful in boundary value problems for Laplace's equation and are much needed in extrapolation theory, vector-valued inequalities and estimates for certain class of non linear differential equation. Muckenhoupt (1970) characterized positive functions w for which the Hardy-Littlewood maximal operator $M$ maps $\mathrm{L}_{\mathrm{p}}\left(\mathrm{R}^{\mathrm{n}}, \mathrm{w}(\mathrm{x}) \mathrm{dx}\right)$ to itself. Muckenhoupt's characterization actually gave the better understanding of theory of weighted inequalities which then led to the introduction of $A_{p}$ class and consequently the development of weighted inequalities [4]. To prove the results, some definition and results are in order:

Definition: The uncentered Hardy-Littlewood maximal operators on $\boldsymbol{R}^{n}$ over balls B is defined as

$$
M(f)(x)=\sup _{x \in B} \operatorname{Avg}|f|=\sup _{x \in B} \frac{1}{|B|} \int_{B}|f(y)| d y .
$$

Similarly the uncentered Hardy-Littlewood maximal operators on $\mathrm{R}^{\mathrm{n}}$ over cubes $\mathrm{Q}$ is defined as

$$
M_{c}(f)(x)=\sup _{x \in Q} \operatorname{Avg}|f|=\sup _{x \in Q} \frac{1}{|Q|} \int_{Q}|f(y)| d y .
$$

In each of the definition above, the suprema are taken over all balls $\mathrm{B}$ and cubes $\mathrm{Q}$ containing the point $\mathrm{X}$. H-L maximal functions are widely used in Harmonic Analysis. For the details about the H-L maximal operators, see [1].

Definition: A locally integrable function on $\boldsymbol{R}^{n}$ that takes values in the interval $(0, \infty)$ almost everywhere is called a weight. So by definition a weight function can be zero or infinity only on a set whose Lebesgue measure is zero.

Definition: A function $\mathrm{w}(\mathrm{x})>0$ is called an $\mathrm{A}_{1}$ weight if there is a constant $\mathrm{C}_{1}>0$ such that

$$
\mathrm{M}(\mathrm{w})(\mathrm{x}) \leq \mathrm{C}_{1} \mathrm{w}(\mathrm{x})
$$


where $\mathrm{M}(\mathrm{w})$ is uncentered Hardy-Littlewood Maximal function given by

$$
M(w)(x)=\sup _{x \in B} \frac{1}{|B|} \int_{B} w(t) d t .
$$

If $\mathrm{w}$ is an $\mathrm{A}_{1}$ weight, then the quantity (which is finite) given by

$$
[w]_{A_{1}}=\sup _{Q \text { cubes inR }}\left(\frac{1}{|Q|} \int_{Q}|w(t)| d t\right)\left\|w^{-1}\right\|_{L^{\infty}(Q)}
$$

is called the $A_{1}$ characteristic constant of $\mathrm{w}$.

Definition: Let $1<\mathrm{p}<\infty$. A weight $\mathrm{w}$ is said to be of class $\mathrm{A}_{\mathrm{p}}$ if $[w]_{A_{p}}$ is finite where $[w]_{A_{p}}$ is defined as

$$
[w]_{A_{p}}=\sup _{Q \text { cubes in } \boldsymbol{R}^{n}}\left(\frac{1}{|Q|} \int_{Q}|w(x)| d x\right)\left(\frac{1}{|Q|} \int_{Q}|w(x)|^{\frac{-1}{p-1}} d x\right)^{p-1} .
$$

We note that in the above definition of $A_{1}$ and $A_{p}$ one can also use set of all balls in $\boldsymbol{R}^{n}$ instead of all cubes in $\boldsymbol{R}^{n}$.

Finally, we state and prove some of the properties of weight functions:

Property 1: Let $w_{1}$ and $w_{2}$ be two $A_{1}$ weights and let $1<\mathrm{p}<\infty$. Then $w_{1} w_{2}{ }^{1-p}$ is an $A_{p}$ weight and $\left[w_{1} w_{2}{ }^{1-p}\right]_{A_{p}} \leq\left[w_{1}\right]_{A_{1}}\left[w_{2}\right]_{A_{1}}^{p-1}$.

Proof: For every cube $\mathrm{Q}$ in $\boldsymbol{R}^{n}$, we have

$$
\begin{aligned}
& \left(\frac{1}{|Q|} \int_{Q} w_{1} w_{2}{ }^{1-p} d x\right)\left(\frac{1}{|Q|} \int_{Q}\left(w_{1} w_{2}{ }^{1-p}\right)^{\frac{-1}{p-1}} d x\right)^{p-1} \\
& \leq\left(\frac{1}{|Q|} \int_{Q} w_{1}\left\|w_{2}^{-1}\right\|_{\mathrm{L} \infty(\mathrm{Q})}^{p-1} d x\right)\left(\frac{1}{|Q|} \int_{Q}\left\|w_{1}^{-1}\right\|_{\mathrm{L} \infty(\mathrm{Q})}^{1 /(p-1)} w_{2} d x\right)^{p-1} \\
& =\left\|w_{2}^{-1}\right\|_{\mathrm{L} \infty(\mathrm{Q})}^{p-1}\left(\frac{1}{|Q|} \int_{Q} w_{1} d x\right)\left\|w_{1}^{-1}\right\|_{\mathrm{L} \infty(\mathrm{Q})}\left(\frac{1}{|Q|} \int_{Q} w_{2} d x\right)^{p-1}
\end{aligned}
$$

Taking supremum on both sides, we have

$$
\begin{aligned}
\sup _{Q}\left[\left(\frac{1}{|Q|} \int_{Q} w_{1} w_{2}{ }^{1-p} d x\right)\left(\frac{1}{|Q|} \int_{Q}\left(w_{1} w_{2}{ }^{1-p}\right)^{\frac{-1}{p-1}} d x\right)^{p-1}\right] \\
\quad \leq \sup _{Q}\left[\left\|w_{2}^{-1}\right\|_{\mathrm{L} \infty(\mathrm{Q})}^{p-1}\left(\frac{1}{|Q|} \int_{Q} w_{1} d x\right)\left\|w_{1}^{-1}\right\|_{\mathrm{L} \infty(\mathrm{Q})}\left(\frac{1}{|Q|} \int_{Q} w_{2} d x\right)^{p-1}\right] \\
\leq \sup _{Q}\left[\left(\frac{1}{|Q|} \int_{Q} w_{1} d x\right)\right]\left\|w_{1}^{-1}\right\|_{\mathrm{L} \infty(\mathrm{Q})} \cdot \sup _{Q}\left[\left(\frac{1}{|Q|} \int_{Q} w_{2} d x\right)^{p-1}\right]\left\|w_{2}^{-1}\right\|_{\mathrm{L} \infty(\mathrm{Q})}^{p-1}
\end{aligned}
$$

This shows that $\left[w_{1} w_{2}{ }^{1-p}\right]_{A_{p}} \leq\left[w_{1}\right]_{A_{1}}\left[w_{2}\right]_{A_{1}}^{p-1}$. Consequently, we have $w_{1} w_{2}{ }^{1-p} \in A_{p}$. Next we state and prove another property: 
Property 2: Let $w_{0} \in A_{p_{0}}$ and $w_{1} \in A_{p_{1}}$ for some $1 \leq p_{0}, p_{1}<\infty$. Let $0 \leq \theta \leq 1$ and define $\frac{1}{p}=\frac{1-\theta}{p_{0}}+$ $\frac{\theta}{p_{1}}$ and $w^{\frac{1}{p}}=w_{0}^{\frac{1-\theta}{p_{0}}} w_{1}^{\frac{\theta}{p_{1}}}$. Then $\mathrm{w}$ is in $A_{p}$ showing that $[w]_{A_{p}} \leq\left[w_{0}\right]_{A_{p_{0}}}^{(1-\theta) \frac{p}{p_{0}}}\left[w_{1}\right]_{A_{p_{1}}}^{\frac{\theta p}{p_{1}}}$.

Proof: Let $p^{\prime}, p_{0}^{\prime}$ and $p_{1}^{\prime}$ be the conjugate exponent of $p, p_{0}$ and $p_{1}$ respectively. We have,

$$
\frac{1}{p}=\frac{1-\theta}{p_{0}}+\frac{\theta}{p_{1}}----------(1)
$$

This gives

$$
1-\frac{1}{p^{\prime}}=(1-\theta)\left(1-\frac{1}{p_{0}{ }^{\prime}}\right)+\theta\left(1-\frac{1}{p^{\prime}}\right)
$$

Simplifying the above relation we get

$$
\frac{1}{p^{\prime}}=\frac{1-\theta}{p_{0}{ }^{\prime}}+\frac{\theta}{p_{1}{ }^{\prime}}-----------(2)
$$

From (1) and (2) we immediately get,

$$
1=\frac{1}{\frac{p_{0}}{1-\theta}}+\frac{1}{\frac{p_{1}}{\theta}} \text { and } 1=\frac{1}{\frac{p_{0}^{\prime}}{1-\theta}}+\frac{1}{\frac{p_{1}^{\prime}}{\theta}} .
$$

Given a cube Q in $\boldsymbol{R}^{n}$, we apply Holder's inequality with the exponent $\frac{p_{0}}{1-\theta}$ and $\frac{p_{1}}{\theta}$ to obtain

$$
\begin{gathered}
\frac{1}{|Q|} \int_{Q} w=\frac{1}{|Q|} \int_{Q} w_{0}^{\frac{(1-\theta) p}{p_{0}}} w_{1}^{\frac{\theta p}{p_{1}}} \\
\leq\left(\frac{1}{|Q|} \int_{Q} w_{0}\right)^{\frac{(1-\theta) p}{p_{0}}}\left(\frac{1}{|Q|} \int_{Q} w_{1}\right)^{\frac{\theta p}{p_{1}}} .
\end{gathered}
$$

These yields

$$
\frac{1}{|Q|} \int_{Q} w \leq\left(\frac{1}{|Q|} \int_{Q} w_{0}\right)^{\frac{(1-\theta) p}{p_{0}}}\left(\frac{1}{|Q|} \int_{Q} w_{1}\right)^{\frac{\theta p}{p_{1}}}
$$

We again apply Holder's inequality with the exponent $\frac{p_{0}{ }^{\prime}}{1-\theta}$ and $\frac{p_{1}{ }^{\prime}}{\theta}$ to obtain

$$
\begin{aligned}
\left(\frac{1}{|Q|} \int_{Q} w^{-\frac{p^{\prime}}{p}}\right)^{\frac{p}{p^{\prime}}} & =\left(\frac{1}{|Q|} \int_{Q} w_{0}^{\frac{-(1-\theta) p^{\prime}}{p_{0}}} w_{1} \frac{-\theta p^{\prime}}{p_{1}}\right)^{\frac{p}{p^{\prime}}} \\
& \leq\left[\left(\frac{1}{|Q|} \int_{Q} w_{0}^{\frac{-p_{0}{ }^{\prime}}{p_{0}}}\right)^{\frac{(1-\theta) p^{\prime}}{p_{0}}}\left(\frac{1}{|Q|} \int_{Q} w_{1}^{\frac{-p_{1}{ }^{\prime}}{p_{1}}}\right)^{\frac{\theta p^{\prime}}{p_{1}}}\right]^{\frac{p}{p^{\prime}}} \\
& =\left(\frac{1}{|Q|} \int_{Q} w_{0}^{\frac{-p_{0}}{p_{0}}}\right)^{\frac{(1-\theta) p}{p_{0}}}\left(\frac{1}{|Q|} \int_{Q} w_{1} \frac{-p_{1}{ }^{\prime}}{p_{1}}\right)^{\frac{\theta p}{p_{1}}}
\end{aligned}
$$




$$
=\left[\left(\frac{1}{|Q|} \int_{Q} w_{0} \frac{-p_{0}{ }^{\prime}}{p_{0}}\right)^{\frac{p_{0}}{p_{0}}}\right]^{\frac{(1-\theta) p}{p_{0}}}\left[\left(\frac{1}{|Q|} \int_{Q} w_{1} \frac{-p_{1}{ }^{\prime}}{p_{1}}\right)^{\frac{p_{1}}{p_{1}^{\prime}}}\right]^{\frac{\theta p}{p_{1}}} .
$$

Consequently we get,

$$
\left(\frac{1}{|Q|} \int_{Q} w^{-\frac{p^{\prime}}{p}}\right)^{\frac{p}{p^{\prime}}} \leq\left[\left(\frac{1}{|Q|} \int_{Q} w_{0} \frac{-p_{0}{ }^{\prime}}{p_{0}}\right)^{\frac{p_{0}}{p_{0}^{\prime}}}\right]^{\frac{(1-\theta) p}{p_{0}}}\left[\left(\frac{1}{|Q|} \int_{Q} w_{1}^{\frac{-p_{1}{ }^{\prime}}{p_{1}}}\right)^{\frac{p_{1}}{p_{1}^{\prime}}}\right]^{\frac{\theta p}{p_{1}}}-----(4)
$$

Now multiplying (3) and (4), we have

$$
\begin{gathered}
\left(\frac{1}{|Q|} \int_{Q} w\right)\left(\frac{1}{|Q|} \int_{Q} w^{-\frac{p^{\prime}}{p}}\right)^{\frac{p}{p^{\prime}}} \leq\left(\frac{1}{|Q|} \int_{Q} w_{0}\right)^{\frac{(1-\theta) p}{p_{0}}}\left[\left(\frac{1}{|Q|} \int_{Q} w_{0}^{\frac{-p_{0}{ }^{\prime}}{p_{0}}}\right)^{\frac{p_{0}}{p_{0}}}\right]^{\frac{(1-\theta) p}{p_{0}}} \\
\times\left(\frac{1}{|Q|} \int_{Q} w_{1}\right)^{\frac{\theta p}{p_{1}}}\left[\left(\frac{1}{|Q|} \int_{Q} w_{1}^{\frac{-p_{1}}{p_{1}}}\right)^{\frac{p_{1}^{\prime}}{p_{1}^{\prime}}}\right]^{\frac{\theta p}{p_{1}}} \\
\leq\left[w_{0}\right]_{A_{p_{0}}}^{(1-\theta) \frac{p}{p_{0}}}\left[w_{1}\right]_{A_{p_{1}}}^{\frac{\theta p}{p_{1}}}
\end{gathered}
$$

Now taking supremum over all cubes $\mathrm{Q}$ in $\boldsymbol{R}^{n}$, we have $w \in A_{p}$.

There are other properties of $A_{p}$ which can be proved using the elementary analysis tools. For more about the weight function and related properties, please refer [2], [3], and [5].

\section{Conclusion}

We studied weight functions and we proved some important properties of these weight functions using the elementary analysis tools.

\section{References}

1. Banelos R. and Moore C.N, "Probabilistic Behavior of Harmonic Functions", Birkhauser Verlag, USA, (1991).

2. Ghimire S., Two Different Ways to Show a Function is an $\mathrm{A}_{1}$ Weight Function, "The Nepali Mathematical Sciences Report", 33(1), (2014).

3. Ghimire S., Weighted Inequality, “Journal of Institute of Engineering”, 10(1), (2014).

4. Grafakos Loukas, “Modern Fourier Analysis”, Second Edition, Springer, (2009).

5. Parajuli V. and Ghimire S., Product of $\mathrm{A}_{\mathrm{p}}$ weight functions, “Journal of Institute of Engineering”, 11(1), (2015). 